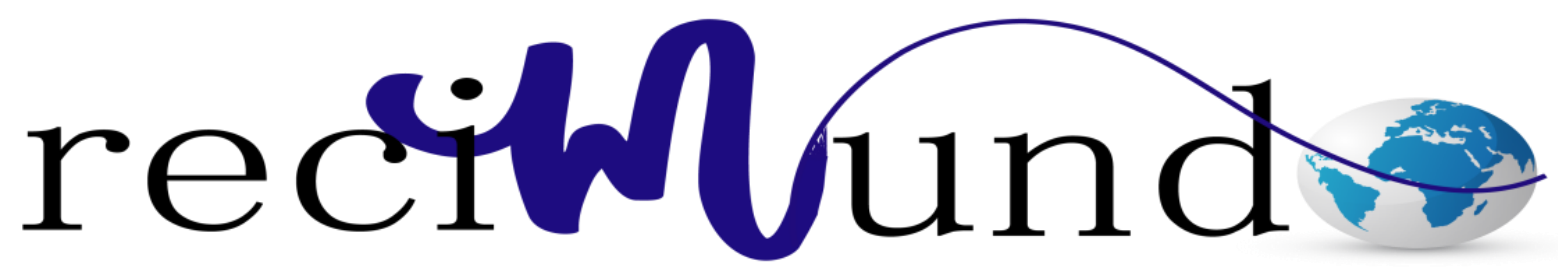

Revista Científica Mundo de la Investigación y el Conocimiento

Shyrle Lisbeth Vaca Rodríguez ${ }^{\text {a }}$; Washington Enrique Zambrano Ordóñez ${ }^{\text {; }}$ José Leonardo Castro Zambrano ${ }^{c}$; Roberto Gabriel Velasquez Arias ${ }^{\text {d }}$

Corrección quirúrgica: reconstrucción de pabellón auricular por microtia vs prótesis de oreja

Surgical correction: reconstruction of auricular pavilion by microtia vs oreja prosthesis

Revista Científica Mundo de la Investigación y el Conocimiento. Vol. 2 núm.3, julio, ISSN: 2588-073X, 2018, pp. 278-286

DOI: 10.26820/recimundo/2.(3).julio.2018.278-286

Editorial Saberes del Conocimiento

Recibido: 05/04/2018

Aceptado: 05/06/2018

Publicado: 30/07/2018

Correspondencia: shyrle.vacar@ug.edu.ec

a. Universidad de Guayaquil; shyrle.vacar@ug.edu.ec

b. Universidad Laica Eloy Alfaro de Manabí; washington.zambranoo@uleam.edu.ec

c. Universidad de Guayaquil; jose.castroz@ug.edu.ec

d. Universidad de Guayaquil; roberto.velasqueza@ug.edu.ec 


\section{Corrección quirúrgica: reconstrucción de pabellón auricular por microtia vs prótesis de oreja}

Vol. 2, núm. 3., (2018)

Shyrle Lisbeth Vaca Rodríguez; Washington Enrique Zambrano Ordóñez; José Leonardo Castro

Zambrano; Roberto Gabriel Velasquez Arias

\section{RESUMEN}

En el siguiente estudio se indagará sobre las correcciones quirúrgicas con respecto a las reconstrucciones de pabellón auricular por microtia versus prótesis de oreja. Primero se tiene que aclarar que el pabellón auricular es una importante unidad estética en el ser humano. Las anomalías de malformación pueden ocurrir de forma aislada o como parte de un síndrome, y pueden ir desde la microtia en distinto grado hasta la anotia. Es importante resaltar que los factores hereditarios y los accidentes vasculares en el útero durante la gestación, se han sugerido como importantes en la etiología de la microtia. La complejidad del pabellón auricular hace que su reconstrucción sea técnicamente dificultosa, debido a las concavidades y convexidades del cartílago subyacente con su adherente y delgada capa de piel que lo recubre. El tratamiento de esta patología es quirúrgico, para esto se han descrito variadas técnicas de reconstrucción. Las más utilizadas son: la reconstrucción con implantes, reconstrucción protésica y la reconstrucción antóloga mediante cartílago costal, siendo esta última la más ampliamente aceptada. Cada una de estas técnicas tiene ciertas indicaciones, así como ventajas y desventajas; asimismo, existen Prótesis (oreja artificial) lo cual es cada vez menos común. Se pudo concluir que el diagnóstico es esencialmente clínico y se basa en el tipo de microtia (unilateral o bilateral), edad del paciente, grado de deformidad, tamaño de la oreja contraria, así como la presencia o ausencia de atresia. Se recomienda que la atención y conducta a tomar en el caso de pacientes con microtia debe ser individualizada, es decir, dependerá de cada paciente el tratamiento a aplicar ya sea corrección quirúrgica (otoplastia) o prótesis de oreja.

Palabras claves: Oreja, pabellón auricular, estética, intervención quirúrgica y prótesis. 


\section{Corrección quirúrgica: reconstrucción de pabellón auricular por microtia vs prótesis de oreja}

Vol. 2, núm. 3., (2018)

Shyrle Lisbeth Vaca Rodríguez; Washington Enrique Zambrano Ordóñez; José Leonardo Castro Zambrano; Roberto Gabriel Velasquez Arias

\section{ABSTRACT}

In the following study, we will investigate the surgical corrections with respect to the reconstruction of the auricular pavilion by microtia versus prosthesis of the ear. First, it must be clarified that the auricular pavilion is an important aesthetic unit in the human being. Malformation abnormalities can occur in isolation or as part of a syndrome, and can range from microtia in varying degrees to anotia. It is important to note that hereditary factors and vascular accidents in the uterus during pregnancy have been suggested as important in the etiology of microtia. The complexity of the auricular pavilion makes its reconstruction technically difficult, due to the concavities and convexities of the underlying cartilage with its adherent and thin layer of skin that covers it. The treatment of this pathology is surgical, for this several techniques of reconstruction have been described. The most used are: reconstruction with implants, prosthetic reconstruction and autologous reconstruction by costal cartilage, the latter being the most widely accepted. Each of these techniques has certain indications, as well as advantages and disadvantages; also, there are Prostheses (artificial ear) which is less and less common. It was concluded that the diagnosis is essentially clinical and is based on the type of microtia (unilateral or bilateral), age of the patient, degree of deformity, size of the opposite ear, as well as the presence or absence of atresia. It is recommended that the attention and behavior to be taken in the case of patients with microtia should be individualized, that is, it will depend on each patient the treatment to be applied either surgical correction (otoplasty) or ear prosthesis.

Keywords: Ear, auricular pavilion, aesthetics, surgical intervention and prosthesis. 


\section{Corrección quirúrgica: reconstrucción de pabellón auricular por microtia vs prótesis de oreja}

Vol. 2, núm. 3., (2018)

Shyrle Lisbeth Vaca Rodríguez; Washington Enrique Zambrano Ordóñez; José Leonardo Castro

Zambrano; Roberto Gabriel Velasquez Arias

\section{Introducción.}

El pabellón auricular es una importante unidad estética en el ser humano. Las anomalías de malformación pueden ocurrir de forma aislada o como parte de un síndrome, y pueden ir desde la microtia en distinto grado hasta la anotia. Se ha reportado una prevalencia que va desde un 0,8 a 2,4 por 10.000 nacidos vivos, siendo menor en población europea y negra que en hispanos y asiáticos.

El pabellón auricular deriva embriológicamente del primer y segundo arco branquial y la patología se produce entre la quinta y sexta semana de gestación debido a múltiples factores tanto genéticos, teratógenos y anormalidades vasculares que influyen en el normal desarrollo. Es importante señalar que el origen embriológico del oído medio e interno es distinto al pabellón auricular, por esa razón es inhabitual la asociación de sordera con malformación auricular, aunque esta sea compleja (Yanine, Giugliano, 2015).

Los factores hereditarios y los accidentes vasculares en el útero durante la gestación, se han sugerido como importantes en la etiología de la microtia. Varios grupos han estudiado a sus pacientes con microtia encontrando evidencia de microsomía craneofacial familiar y patrones sugerentes de herencia multifactorial. Cuando la microtia se asocia a atresia o a ausencia del meato auditivo externo (con disminución de la agudeza auditiva), sugiere una detención del desarrollo. La oreja se forma del primer arco branquial (mandibular) y del segundo (hioideo); comienza su desarrollo a partir de la $5^{\mathrm{a}}$ semana de gestación, en cualquier lado de la primera hendidura branquial entre estos 2 arcos, lo que se convierte en el canal externo. 


\section{Corrección quirúrgica: reconstrucción de pabellón auricular por microtia vs prótesis de oreja}

Vol. 2, núm. 3., (2018)

Shyrle Lisbeth Vaca Rodríguez; Washington Enrique Zambrano Ordóñez; José Leonardo Castro Zambrano; Roberto Gabriel Velasquez Arias

Finalmente, el primer arco contribuye al trago y a la raíz del hélix y el resto de la oreja se forma del segundo arco. Inicialmente, la oreja tiene una posición ventromedial, que llega a ser más dorsolateral en el tercio medio facial, hasta que los procesos mandibulares crecen y la empujan hacia fuera y hacia arriba. La interrupción en la proliferación de estas etapas del desarrollo puede producir las estructuras rudimentarias variables que se presentan como microtia. La anatomía de la oreja micrótica es similar a la del embrión de 6 semanas de vida. (Sesman, Carmona, Herrera, Fichtl, León, 2011)

La complejidad del pabellón auricular hace que su reconstrucción sea técnicamente dificultosa, debido a las concavidades y convexidades del cartílago subyacente con su adherente y delgada capa de piel que lo recubre. En contraste a muchas otras estructuras faciales, las orejas no son consideradas un objeto de belleza independientemente admirado. El común de las personas encontrará dificultoso dibujar un pabellón auricular de memoria; sin embargo, ellos podrían fácilmente indicar si existiese una prominencia excesiva o una asimetría en la oreja. El objetivo de la reconstrucción auricular, por lo tanto, es recrear la forma y topografía de una oreja para hacerla "imperceptible" a la vista, más que una estructura hermosa (Schulz, Salem, 2003).

El tratamiento de esta patología es quirúrgico, para esto se han descrito variadas técnicas de reconstrucción. Las más utilizadas son: la reconstrucción con implantes, reconstrucción protésica y la reconstrucción autóloga mediante cartílago costal, siendo esta última la más ampliamente aceptada. Cada una de estas técnicas tiene ciertas indicaciones, así como ventajas y desventajas; asimismo, existen Prótesis (oreja artificial) lo cual es cada vez menos común. (Yanine, Giugliano, 2015). 


\section{Corrección quirúrgica: reconstrucción de pabellón auricular por microtia vs prótesis de oreja}

Vol. 2, núm. 3., (2018)

Shyrle Lisbeth Vaca Rodríguez; Washington Enrique Zambrano Ordóñez; José Leonardo Castro

Zambrano; Roberto Gabriel Velasquez Arias

\section{Materiales y métodos.}

En el presente trabajo se recurrió a la investigación documental como metodología de estudio para llevar adelante este documento. Esta estrategia metodológica consiste en la observación y reflexión sistemática de realidades teóricas y empíricas, en las que se usa una gran variedad de documentos, con la finalidad de indagar, interpretar, exponer cifras e informaciones referentes al tema de ciencia en el cual se está trabajando. En esta labor se utilizan herramientas que tienen como fin conseguir resultados que pueden ser la plataforma para otra exploración científica o la construcción de nuevos conocimientos. (Alfonzo, 1994)

La investigación documental tiene la particularidad de utilizar como una fuente primaria de insumos, más no la única y exclusiva, el documento escrito en sus diferentes formas: documentos impresos, electrónicos y audiovisuales. Las fuentes impresas incluyen: libros enciclopedias, revistas, periódicos, diccionarios, monografías, tesis y otros documentos. Asimismo, se pueden usar fuentes electrónicas como: correos electrónicos, CD roms, base de datos, revistas y periódicos en línea y páginas web. (Sampieri, 1998)

El trabajo a realizar con este método se puede enumerar de la siguiente forma: realizar un proceso de abstracción científica, generalizando sobre la base de lo fundamental; utilizar los procedimientos lógicos y mentales de toda investigación; tales como: análisis, síntesis, deducción, inducción, entre otros; y se debe a una recopilación adecuada de datos, que permiten redescubrir hechos, sugerir problemas, ubicar hacia otras fuentes de investigación, orientar formas para elaborar instrumentos de investigación y elaborar hipótesis (Rodríguez, 2013) 


\section{Corrección quirúrgica: reconstrucción de pabellón auricular por microtia vs prótesis de oreja}

Vol. 2, núm. 3., (2018)

Shyrle Lisbeth Vaca Rodríguez; Washington Enrique Zambrano Ordóñez; José Leonardo Castro Zambrano; Roberto Gabriel Velasquez Arias

Con lo expuestos se puede asegurar que en el presente trabajo de investigación se utilizó como metodología una revisión bibliográfica documental no experimental, puesto que se realizó un análisis de diferentes artículos médicos y de opiniones referentes, siempre privilegiando las publicaciones más recientes y con mayor prestigio, sobre las correcciones quirúrgicas con respecto a las reconstrucciones de pabellón auricular por microtia versus prótesis de oreja.

\section{Resultados.}

La otoplastia con expansión tisular local permite disponer de tejido en cantidad, color y textura similares al auricular. En su asociación con la prótesis de poliuretano se logra una arquitectura auricular ideal, con un surco retroauricular bien delimitado y sin necesidad de injertos de cartílago y/ o pie. (Iwanyk, Iacouzzi., 2007).

Las prótesis auriculares conforme se ha ido avanzando con éxito en la reconstrucción de la oreja, es cada vez menos común elegir la prótesis a menos de que no haya otra opción. En algunas ocasiones, por alguna razón es imposible hacer las otras reconstrucciones. Las prótesis están hechas de un material suave (silicón), y se crean con impresiones de otras orejas y pintadas a mano para que se acerquen lo más posible al tono de piel (Infogen, 2018).

La reconstrucción auricular es reconfortante en la medida en que se prepare de manera adecuada el cirujano plástico para enfrentar dicho problema. Las campañas de cirugía extramuros proporcionan una posibilidad única de unir los factores antes mencionados en el perfeccionamiento de una técnica seleccionada (Viera, Valero, 2007). 


\section{Corrección quirúrgica: reconstrucción de pabellón auricular por microtia vs prótesis de oreja}

Vol. 2, núm. 3., (2018) Shyrle Lisbeth Vaca Rodríguez; Washington Enrique Zambrano Ordóñez; José Leonardo Castro Zambrano; Roberto Gabriel Velasquez Arias

\section{Conclusiones.}

Todo cirujano se verá enfrentado en algún momento a una lesión del pabellón auricular, sea traumática, oncológica o congénita. Es importante tener claros los conceptos que se refieren a la reconstrucción auricular de los diferentes defectos para adoptar conductas adecuadas y decidir cuándo puede el médico general o el cirujano reparar por sus medios o cuándo debe derivar al cirujano plástico reparador, en busca de un mejor resultado estético para el paciente. Siempre hay que tener presente que el objetivo en la reconstrucción auricular es priorizar la imperceptibilidad de la oreja por sobre una recreación exacta de su forma y relieve. (Schulz, Salem, 2003).

El diagnóstico es esencialmente clínico y se basa en el tipo de microtia (unilateral o bilateral), edad del paciente, grado de deformidad, tamaño de la oreja contraria, así como la presencia o ausencia de atresia (Viera, Valero, 2007)

\section{Recomendaciones.}

La atención y conducta a tomar en el caso de pacientes con microtia debe ser individualizada, es decir, dependerá de cada paciente el tratamiento a aplicar ya sea corrección quirúrgica (otoplastia) o prótesis de oreja, siempre tomando en cuenta las características físicas y clínicas de cada individuo.

Si el paciente se detecta al nacimiento, lo que es usual, y si el tipo de microtia es bilateral, el diagnóstico se inicia de manera temprana antes de los 4 meses de vida con la realización de una prueba de respuesta cerebral auditiva, la cual da una idea clara de las capacidades sensoriales de audición y del porcentaje exacto de pérdida conductiva presente (Viera, Valero, 2007). 


\section{Corrección quirúrgica: reconstrucción de pabellón auricular por microtia vs prótesis de oreja}

Vol. 2, núm. 3., (2018)

Shyrle Lisbeth Vaca Rodríguez; Washington Enrique Zambrano Ordóñez; José Leonardo Castro Zambrano; Roberto Gabriel Velasquez Arias

\section{Bibliografía}

1. Alfonzo, I. (1994). Técnicas de investigacion bibliografia. Caracas: Contexto Ediciones.

2. Infogen. (2018). Microtia, oreja pequeña. México. Infogen. Recuperado de http://infogen.org.mx/microtia-oreja-pequena/

3. Iwanyk, P., \& Iacouzzi., S. (2007). Innovación en la reconstrucción del pabellón auricular disgenésico con tejido expandido e implante. Cirugía Plástica IberoLatinoamericana,33(2), 123-128.

4. Rodríguez, M. (2013). Acerca de la Investigacion bibliografica y documental. Guia de Teses. Recuperado de http://guiadetesis. wordpress. com/2013/08/19/acerca-de-lainvestigacion-bibliografica-ydocumental

5. Sampieri, R. (1998). Metodologia de la Investigacion. Mexico DF: Interamericana editores.

6. Schulz, M., Salem, C. ( 2003). Reconstrucción auricular . Cuaderno de Cirugía, 17, 7585.

7. Sesman, A., Carmona, R., Herrera, A., Fichtl, A., León, J., Fernández S. (2011). Reconstrucción auricular con implantes osteointegrados en un solo tiempo en pacientes seleccionados. Cirugía Plástica Ibero-Latinoamericana, 37(3), 267-279. Recuperado de http://scielo.isciii.es/scielo.php?script=sci_arttext\&pid=S0376-78922011000300008

8. Viera, M., Valero, U. (2004). Reconstrucción tridimensional de la oreja micrótica. Cirugia Plastica, 14(1), 27-34. Recuperado de http://www.medigraphic.com/pdfs/cplast/cp-2004/cp041e.pdf

9. Yanine, J., Giugliano, C. (2015). Microtia: definición, clasificación y tratamiento. Revisión de la literatura. Revista Pediatría Electrónica, 12(3),13-18. 